\title{
Factors that Affect Ion Trap Data-Dependent MS/MS in Proteomics
}

\author{
Brett R. Wenner and Bert C. Lynn \\ Department of Chemistry, University of Kentucky, Lexington, Kentucky, USA
}

\begin{abstract}
Quadrupole ion trap scanning parameters for performing bottom-up proteomics in a datadependent fashion were evaluated on a Finnigan LCQ Deca mass spectrometer. Evaluation of parameters such as the number of averaged full scans, the number of averaged MS/MS scans, and ion injection times were necessary for acquiring high quality MS/MS spectra that yield favorable $b$ and $y$ ion coverage and high correlation to proteins using database searching algorithms. In this study, we demonstrated how the duty cycle of the mass spectrometer affects the number of peptides that can be successfully identified by SEQUEST using a model system of tryptic BSA peptides to mimic a typical complex mixture associated with bottom-up proteomics. The number of averaged scans and the duration of ion accumulation in the trap had a significant effect on the quality of acquired MS/MS spectra. For example, by increasing the ion injection time from $500 \mathrm{~ms}$ to $600 \mathrm{~ms}$, peptide HLVDEPQNLIK improved from being improperly identified to being correctly identified with a SEQUEST cross-correlation score of 3.60. As a result of these experiments, we have devised the following set of ion trap parameters for performing bottom-up proteomics analysis in our laboratory: Three averaged full scans, five averaged MS/MS scans, and a maximum ion injection time of $600 \mathrm{~ms}$. (J Am Soc Mass Spectrom 2004, 15, 150-157) (c) 2004 American Society for Mass Spectrometry
\end{abstract}

I n recent years, the bottom-up approach to proteomics has gained much notoriety in the scientific community [1-15]. This approach begins with the proteolysis of a protein or mixture of proteins to generate an even more complex mixture of peptides. These peptides are isolated, subjected to direct MS/MS analysis, and identified via protein database searching algorithms that match the acquired MS/MS spectra to computer-predicted spectra and lead to the identification of the parent proteins [16, 17]. However, the challenge of accurately identifying all proteins present in a mixture relies on the ability to sufficiently resolve all peptides resulting from the enzymatic digestion while simultaneously acquiring high quality MS/MS spectra which will lead to proper ion assignments and protein correlations by the database searching algorithm.

To address the problem of resolving such complex mixtures of peptides, some research groups have employed multidimensional HPLC techniques [6-15, 1821]. In these schemes, peptides are separated by utilizing two or more of their physical properties such as hydorophobicity, charge, and size. Moving to multiple dimensions of separation modes increases peak capacity (i.e., the maximum number of components that can

Published online December 4, 2003

Address reprint requests to Dr B. C. Lynn, Department of Chemistry, University of Kentucky, Chemistry/Physics Building, Lexington, KY 405060286, USA. E-mail: bclynn2@uky.edu be resolved over a given separation length). An increase in chromatographic peak capacity translates into a greater number of peptides that can be resolved and thus interrogated by MS/MS analysis. Multidimensional separations undoubtedly have greatly increased the number of proteins that can be identified in complex mixtures; however, these techniques do have their limitations, and peak capacity is still an issue.

Bottom-up proteomics on complex mixtures would not be possible without the existence of instrument decision-making algorithms that can automatically perform MS/MS experiments on precursor ions selected from a previously acquired full scan. The use of such algorithms is widely known as data-dependent analysis. [Note: "Data-dependent" analysis is often associated with the Intelligent Data-Dependent tools of Thermo Finnigan (San Jose, CA) instruments. This term is analogous to SmartSelect ${ }^{\mathrm{TM}}$ (Bruker Daltonics, Inc., Billerica, MA) and Information Dependent Acquisition (Applied Biosystems, Foster City, CA).] This feature enables the instrument to make real-time decisions concerning the experiment at hand. For example, as peptides are eluted from an HPLC separation, the mass spectrometer continuously acquires full scan data. Once an ion is detected above a preset threshold, the instrument automatically switches from full scan to MS/MS mode to perform a tandem experiment on that ion. In the event of multiple, coeluting peptides, the instrument will perform a MS/MS analysis on one ion (usually the most intense), place that ion on an exclu- 
sion list, and then continue on with its data-dependent analysis of other ions present in the full scan. Once an ion is subjected to MS/MS and placed on the exclusion list, it remains there and will not be polled again for a user-defined length of time. The time required for the mass spectrometer to perform a cycle of one analytical full scan followed by one analytical MS/MS scan is dictated by the duty cycle of the instrument. For ion trap instruments, one analytical scan can be composed of a number of averaged scans. Averaging multiple scans to build one analytical scan is necessary to improve the ion statistics achieved by the ion trap mass analyzer. However, increasing the number of averaged scans reduces the duty cycle of the instrument, thus reducing the frequency of acquiring analytical scans. This, in turn, results in fewer MS/MS experiments that can be conducted over a given period of time. In cases where poor chromatographic resolution generates several overlapping peptides, fewer analytical scans per unit time may prevent some peptides from being subjected to an MS/MS experiment. The frequency of analytical scan acquisition is influenced by instrumental parameters such as the number of averaged full scans, the number of averaged MS/MS scans, and ion trap injection time.

In our laboratory, we utilize a micro-flow liquid chromatographic (micro-LC) system for the proteomic analysis of digested protein mixtures. In this setup, we utilize $320 \mu \mathrm{m}$ i.d. capillary columns and flow rates on the order of $4 \mu \mathrm{L} / \mathrm{min}$. This arrangement provides us with the reliability we need to analyze large volumes of samples of unknown quality and/or origin. Although micro-LC may be slightly less sensitive, its robustness far surpasses that of nano-flow (200-300 nL/min) systems. Nano-LC setups are commonly subject to plugging due to their small column and emitter innerdiameters, making the analysis of "real world" samples a challenge. Additionally, when dealing with $\mathrm{nL} / \mathrm{min}$ flow rates, dead volumes become a greater concern. This issue is typically dealt with by using trapping columns. By first loading the sample onto a trapping column at higher flow rates (i.e., $\mu \mathrm{L} / \mathrm{min}$ regime), then switching to nano-flow for the analytical separation, long sample loop evacuation times are avoided. This, however, normally requires multiple LC pumps and valving/switching capabilities.

To perform successful bottom-up proteomics, a delicate balance between peak capacity, analytical scan acquisition rate, and ion abundances must be achieved. Ion abundance is largely dictated by the amount of protein present in the sample. In most "real world" samples, it is impossible to optimize the quantities of all proteins present. Therefore, the majority of effort must be spent optimizing peak capacity and the analytical scan acquisition rate. As mentioned previously, great advancements in increasing peak capacity have been made with the development of multidimensional techniques. Now we have the capability to resolve orders of magnitude more peptides than was possible with tra- ditional one-dimensional LC techniques. A large number of laboratories are currently using quadrupole ion traps for proteomics research. Recently, the Proteomics Research Group (PRG) of the Association of Biomolecular Research Facilities (ABRF) conducted an open study in which research laboratories were invited to participate [22]. The study required the participants to identify the sequences and phosphorylation sites of a peptide mixture. A total of 57 different laboratories participated in this study. Of these, $39 \%$ of the laboratories utilized ion trap mass analyzers, attesting to the utility of ion traps in proteomics. However, to date, very little consideration has been given to the optimization of ion trap parameters for proteomics applications. Parameters such as the number of averaged scans (full scan and MS/MS) and the injection time for ion accumulation play a critical role in achieving high quality spectra. Simultaneous optimization of all three elements is crucial to achieve high quality MS/MS spectra of all peptides present in a sample. This, in turn, will result in more accurate fragment ion assignments by database searching algorithms leading to higher protein correlations.

In this work, we investigate the scan parameters of one variety of ion trap mass analyzer, the LCQ Deca. Furthermore, we report the effects of these parameters on the data-dependent algorithm and our ability to analyze the maximum number of closely eluting peptides. The balance between ion trap scan parameters and the ability to maintain an analytical scan acquisition rate amenable to interrogating the maximum number of peptides are explored. We present these experimental parameters and their influence on the quality of MS/MS data.

\section{Experimental}

\section{Materials}

Bovine serum albumin (BSA), ammonium acetate $\left(\mathrm{NH}_{4} \mathrm{HCO}_{3}\right)$, and ammonium bicarbonate were obtained from Sigma Chemical Co. (St. Louis, MO). HPLC grade water, HPLC grade acetoniltrile (ACN), and formic acid were purchased from Fisher Scientific (Fair Lawn, NJ). Sequencing grade modified trypsin was a product of Promega (Madison, WI).

\section{Digestion of $B S A$}

A stock solution of $2 \mu \mathrm{g} / \mu \mathrm{L}$ BSA was prepared in 50 $\mathrm{mM} \mathrm{NH} \mathrm{NHO}_{3}$. A $50 \mu \mathrm{L}$ aliquot was removed and mixed with $50 \mu \mathrm{L}$ of ACN to improve proteolysis [23]. Trypsin was added to a final protease-to-BSA ratio of $1: 40$, and the digestion mixture was incubated at $37^{\circ} \mathrm{C}$ for $24 \mathrm{~h}$. The resulting digested BSA sample was used to prepare 10-, 100-, and 1000-fold serial dilutions in water (i.e., protein concentrations of 100,10 , and $1 \mathrm{ng} / \mu \mathrm{L}$, respectively). 


\section{Chromatography and Mass Spectrometry}

Digested BSA samples were resolved on a $15 \mathrm{~cm} \times 320$ $\mu \mathrm{m}$ i.d. reverse phase capillary column. This column was fabricated in-house and packed with $5 \mu \mathrm{m}$ Macrosphere 300 C18 particles (Alltech Associates, Inc., Deerfield, IL) using a stainless steel packing cell pressurized with helium to 1000 psi. Sample introduction and mobile phase delivery at $4 \mu \mathrm{L} / \mathrm{min}$ was performed by a LC Packings Ultimate capillary LC system (San Francisco, CA) equipped with a $5-\mu \mathrm{L}$ injection loop. Mobile phase A was water $+0.1 \%$ formic acid and mobile phase B was ACN $+0.1 \%$ formic acid. A linear gradient of $5-95 \%$ B over 55 min was employed for the separation of the BSA peptides.

Eluting peptides in the column effluent were directly electrosprayed into a Finnigan LCQ Deca ion trap mass spectrometer (Thermo Finnigan, San Jose, CA) for analysis. The electrospray source was given $4.5 \mathrm{kV}$ of applied voltage and 33 arbitrary units (range $0-100$ ) of sheath gas flow, while the inlet capillary was held at $19.5 \mathrm{~V}$ and $170{ }^{\circ} \mathrm{C}$. Other mass spectrometer parameters were as follows: tube lens offset at $-15.0 \mathrm{~V}$, multipole 1 offset at $-5.3 \mathrm{~V}$, multipole 2 offset at $-11.0 \mathrm{~V}$, intermultipole lens at $-77.0 \mathrm{~V}$, entrance lens at $-82.0 \mathrm{~V}$ and trap DC offset at $-10.0 \mathrm{~V}$. Spectra of eluting BSA peptides were acquired in a data-dependent fashion by first acquiring a full MS scan from $\mathrm{m} / \mathrm{z} 150$ to 2000 followed by a MS/MS scan between $\mathrm{m} / \mathrm{z} 150$ and 2000 of the most intense ion of the previous full MS scan. MS/MS scans were acquired using an activation $\mathrm{q}_{\mathrm{z}}$ of 0.250 , activation time of $30 \mathrm{~ms}$, and $35 \%$ normalized collision energy (NCE). (Note: NCE, is the amplitude of the resonance excitation RF voltage scaled to the precursor mass based on the formula: RF amplitude $=[\mathrm{NCE} \% / 30 \%]$ (precursor ion mass $\times$ tick amp slope + tick amp intercept), where the tick amp slope and tick amp intercept are instrument specific values. For our LCQ Deca, 35\% NCE for $m / z 1000=1.8$ V.) Once sampled, each MS/MS precursor mass was excluded from further tandem experiments for three min. For each analytical full MS and MS/MS scan, $m$ number of MS scans and $n$ number of MS/MS scans were averaged, respectively. In these experiments, $5-\mu \mathrm{L}$ injections of the 10-fold dilution (100 $\mathrm{ng} / \mu \mathrm{L}$ ) of digested BSA were analyzed 25 times; each time $m$ and $n$ were assigned a value of $1,3,5,7$, or 9 averaged scans until a five-by-five experimental array had been acquired. To further refine the impact of sample concentration on the quality of MS/MS scans, similar experiments were performed on the 100 -fold (10 $\mathrm{ng} / \mu \mathrm{L})$ and $1000-$ fold $(1 \mathrm{ng} / \mu \mathrm{L})$ dilutions of digested BSA; however, in these cases, only 1,5, and 9 MS and MS/MS averaged scans were investigated to produce a three-by-three array of data.

A flow injection experiment was also performed in which the reverse phase capillary column was removed and replaced with $50 \mu \mathrm{m}$ i.d. PEEK tubing. Mobile phase $(5 \% \mathrm{ACN})$ was delivered at $4 \mu \mathrm{L} / \mathrm{min}$ to the electrospray source of the mass spectrometer as before.
BSA digest $(10 \mathrm{ng} / \mu \mathrm{L})$ was introduced into the flow in $5-\mu \mathrm{L}$ injections. As the sample plug was sprayed into the mass spectrometer, spectra were again collected in a data-dependent manner for a time period of one min. For this experiment, injections were repeated in triplicate for $n=1,3,5,7$, and 9 averaged MS/MS scans, while the number of averaged full scans was held constant at $m=3$. In all experiments, the automatic gain control (AGC) of the instrument was turned on with target values of $5.0 \times 10^{7}$ and $2.0 \times 10^{7}$ for the MS and MS/MS scans, respectively. For the 10-fold dilution studies, the maximum ion injection times were set at $300 \mathrm{~ms}$ for the full scans and $500 \mathrm{~ms}$ for the MS/MS scans. For all other experiments, the maximum MS/MS ion injection time was increased to $600 \mathrm{~ms}$.

To evaluate the quality of data acquired, the MS/MS spectra were searched against the NCBI non-redundant database using the SEQUEST algorithm to generate cross-correlation scores for each peptide. However, in each experiment, attention was only given to the spectra acquired within a five-min window (approximately $t=$ 21.5-26.5 min) where several peptides were coeluting. The resulting cross-correlation scores (Xcorrs) were subjected to criteria as set by Washburn, et al. [8]. In short, $+1,+2$, and +3 charged peptides had to be tryptic in nature and have cross-correlation scores of at least $1.8,2.2$, and 3.75 , respectively. BSA peptide correlations not meeting these criteria were considered to have ambiguous identifications.

\section{Results and Discussion}

In these experiments, we explored various ion trap parameters (i.e., number of averaged full scans, number of averaged MS/MS scans, and ion injection times) capable of sampling as many poorly resolved peptides as possible, while maintaining high quality MS/MS spectra. A typical reverse phase separation of tryptic BSA peptides is shown in Figure 1. shaded area denotes the five-min time window that was considered for this study. In this window, several peptides coeluted with overlapping peaks. We focused only on these peptides because we wished to mimic very complex mixtures of peptides and to tax the instrument's data-dependent function.

Analysis of the 10-fold diluted BSA digest at the various numbers of averaged scans ( $m$ and $n=1,3,5$, 7 , and 9) yielded a five-by-five matrix of 25 total runs. To understand the effect that the number of averaged scans had on the instrument's frequency of acquiring analytical scans, we looked at the number of MS/MS spectra acquired during the five-min window of interest for each run (Table 1). The number of MS/MS spectra varied from as many as $139(m=1$ full scan, $n$ $=1 \mathrm{MS} / \mathrm{MS}$ scan $)$ to as few as 26 ( $m=9$ full scans, $n=$ 9 MS/MS scans). Further investigation showed that the average instrumental processing time required to acquire one analytical full scan followed by one analytical MS/MS scan ranged from $1.9 \mathrm{~s} \mathrm{(} m=1$ full scan, $n=1$ 


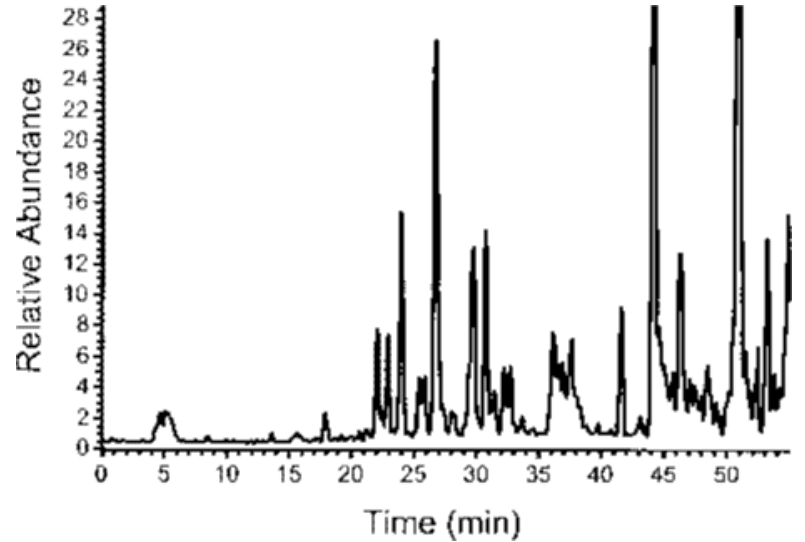

Figure 1. A chromatogram depicting a typical reverse phase separation of BSA tryptic peptides. The shaded region represents the five-min window of interest and contains several poorly resolved, coeluting peptides. Only peptides eluting in this region were evaluated in all experiments.

MS/MS scan) to $15.4 \mathrm{~s}$ ( $m=9$ full scans, $n=9 \mathrm{MS} / \mathrm{MS}$ scans). This illustrated how the frequency of acquiring analytical scans affected the number of spectra that could be acquired over a given time. As we increased the total number of averaged scans that had to be acquired and averaged, more time was required to generate one analytical scan, which in turn resulted in a fewer number MS/MS spectra that could be obtained. For this reason, fewer number of analytical scans per second would be undesirable in situations where several peptides are coeluting.

To better understand the relationship between the number of scans that were averaged and spectra quality, we performed database searches of the MS/MS spectra acquired during the five-min window of interest for all 25 combinations of averaged scans. Only those spectra yielding unambiguous peptide matches

Table 1. Number of MS/MS spectra acquired at various combinations of averaged full scans and MS/MS scans.

Number of Averaged MS/MS Scans

\begin{tabular}{|c|c|c|c|c|c|}
\hline \multirow{3}{*}{$\begin{array}{ll}0 & 1 \\
\stackrel{0}{0} & \\
0 & \\
\bar{D} & 3\end{array}$} & 1 & 3 & 5 & 7 & 9 \\
\hline & 139 & 81 & 60 & 46 & 38 \\
\hline & 100 & 67 & 51 & 42 & 34 \\
\hline तु & 73 & 57 & 46 & 36 & 30 \\
\hline $\begin{array}{l}\overline{0} \\
\bar{d}\end{array}$ & 62 & 49 & 39 & 33 & 27 \\
\hline 9 & 54 & 40 & 34 & 30 & 26 \\
\hline
\end{tabular}

Table 2. Number of unique peptides unambiguously identified from $500 \mathrm{ng}$ of BSA digest at various combinations of averaged full scans and MS/MS scans.

\section{Number of Averaged MS/MS Scans}

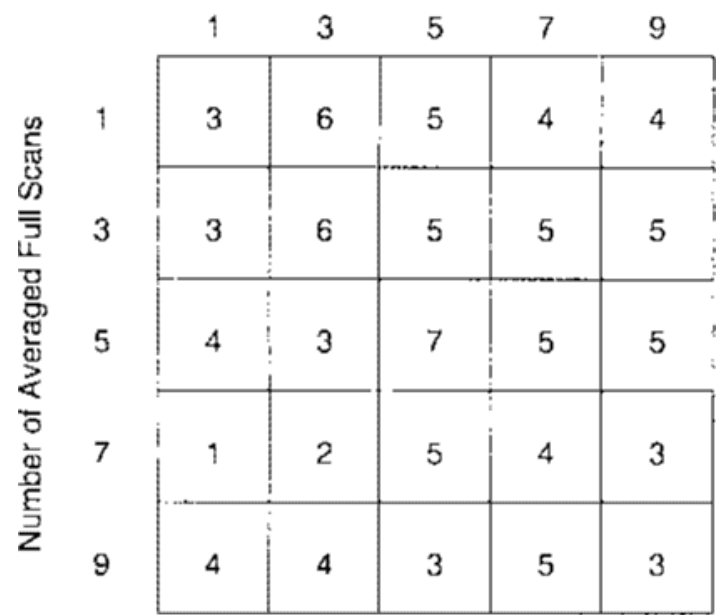

were considered. The numbers of unique BSA peptides identified for each combination of averaged scans were tabulated (Table 2). This set of experiments yielded as few as one and as many as seven unique peptide matches. However, when closely examined, the data revealed an area of enriched peptide matches. This area was bracketed by the ion trap settings of $m=1$ full scan, $n=3 \mathrm{MS} / \mathrm{MS}$ scans and $m=5$ full scans, $n=9 \mathrm{MS} / \mathrm{MS}$ scans. The runs encompassed by these settings produced mostly 5, 6, or 7 BSA peptide matches. It was also noted that the maximum MS/MS ion injection time of $500 \mathrm{~ms}$ was entirely utilized for almost all of the acquired MS/MS scans.

We then decreased the amount of injected sample by a factor of 10 (50 $\mathrm{ng}$ on column) in an attempt to further target a combination of averaged scans that would be superior to the others in identifying the greatest number of unique peptides. This time, however, the experimental array was reduced to nine individual runs consisting of combinations of 1,5, and 9 averaged scans to

Table 3. Number of unique peptides identified from $50 \mathrm{ng}$ of BSA digest at various combinations of averaged full scans and MS/MS scans.

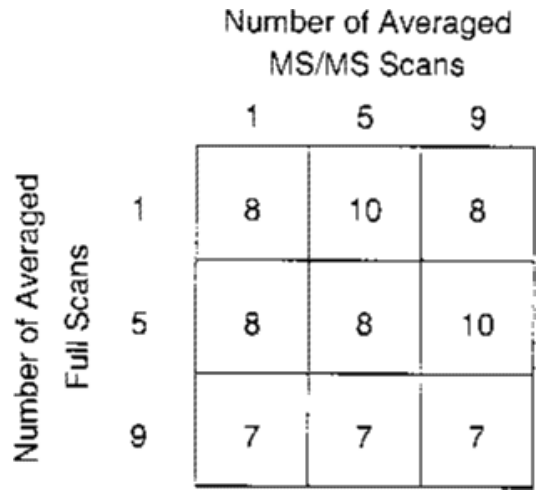




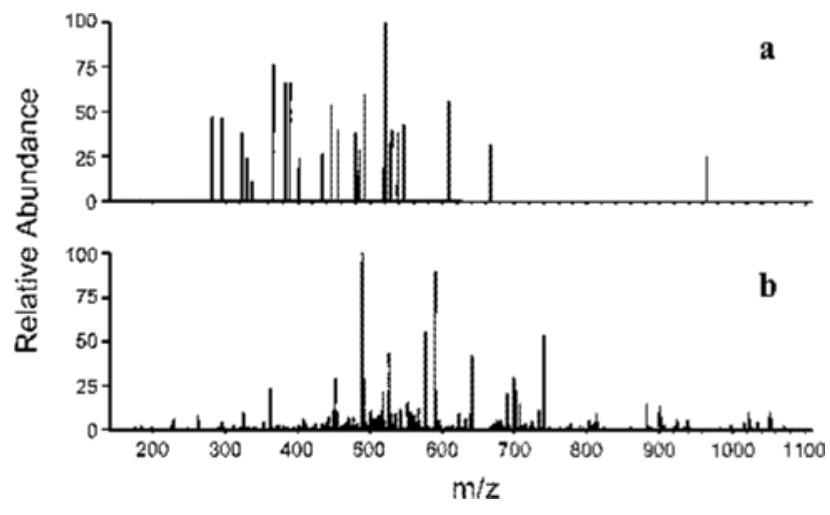

Figure 2. MS/MS spectra of BSA peptide KVPQVSTPTLVEVSR $(+3)$ at $547.7 \mathrm{~m} / \mathrm{z}$. (a) $500 \mathrm{ng}$ of BSA digest with a maximum ion injection time of $500 \mathrm{~ms}$. (b) $50 \mathrm{ng}$ of BSA digest with a maximum ion injection time of $600 \mathrm{~ms}$.

expedite the experimental process. In addition, the maximum MS/MS ion injection time was increased from $500 \mathrm{~ms}$ to $600 \mathrm{~ms}$. This resulted in a surprisingly large increase of identified peptides across the board despite the decrease in sample concentration (Table 3). Close examination of the MS/MS spectra revealed an overall increase in spectral quality, including increases in total ion intensities and $b$ and $y$ fragment ion coverage. This was especially pronounced in +3 charged peptides (Figure 2), the previously bracketed combinations of averaged scans ( $m=1$ full scan, $n=3 \mathrm{MS} / \mathrm{MS}$ to $m=5$ full scans, $n=9 \mathrm{MS} / \mathrm{MS}$ ) seemed to once again generate higher numbers of matched peptides.

To further investigate this, a third array of experiments was conducted using a 1000-fold dilution of stock BSA digest (5 ng on column; Table 4). Again, the array was truncated to a three-by-three scan matrix using maximum MS/MS ion injection times of $600 \mathrm{~ms}$. Searching these acquisitions against the database produced matched peptide numbers similar to those achieved with $500 \mathrm{ng}$ of sample on column with maximum MS/MS injection times of $500 \mathrm{~ms}$. Once again, an area of enhanced peptide correlation was observed in the upper right hand quadrant of the table as bracketed

Table 4. Number of unique peptides identified from $5 \mathrm{ng}$ of BSA digest at various combinations of averaged full scans and MS/MS scans.

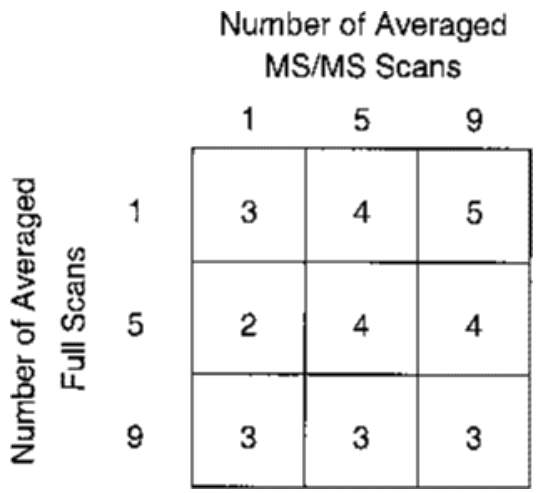

by $m=1$ full scan, $n=3$ MS/MS scans and $m=5$ full scans, $n=9 \mathrm{MS} / \mathrm{MS}$ scans.

The impact that the ion injection times had on the number of identified BSA peptides was quite remarkable and warranted further investigation. To do this, we held the combination of averaged full scans and MS/MS scans at 3 and 5, respectively. Triplicate analyses of the 1000-fold dilution of BSA digest were performed at maximum ion injection times of 500, 600, and $700 \mathrm{~ms}$. To evaluate the effect that injection times had on the quality of acquired MS/MS spectra, we looked at the average Xcorr of four unique BSA peptides (Table 5). In all cases, the MS/MS spectra acquired with 600-ms maximum injection times provided correctly assigned peptide sequences and yielded the best Xcorrs. Acquisitions at 500- and 700-ms maximum injection times, however, produced statistically lower Xcorrs and occasionally generated SEQUEST search results that were not the proper amino acid sequences for these peptides. Examples of MS/MS spectra acquired at each ion injection time for the BSA peptide HLVDEPQNILK are shown in Figure 3. 1ts indicated that a maximum ion injection time of $500 \mathrm{~ms}$ was not sufficiently long to fill the ion trap with a suitable population of precursor ions. In this case few fragment ions were generated, contributing to low total ion current, thus providing poor MS/MS spectra quality. On the other hand, $700 \mathrm{~ms}$ may have been too long to accumulate ions in the trap, resulting in space charge effects. It should be noted that, in most MS/MS scans, all $700 \mathrm{~ms}$ were utilized for ion accumulation, which may have been an indication that the AGC target value of $2.0 \times 10^{7}$ was too high.

A flow injection experiment was performed in an attempt to further isolate an ideal set of scan parameters. Sample introduction of $10 \mathrm{ng} / \mu \mathrm{L}$ BSA digest was made in multiple $5-\mu \mathrm{L}$ injections. As each sample plug was sprayed into the mass spectrometer, the instrument acquired as many data-dependent spectra as possible in one min, the elution time of a typical chromatographic peak (i.e., full width at base). For each injection, the number of full scans were held constant at $m=3$, while the number of averaged MS/MS scans were set at $n=$ $1,3,5,7$, or 9 . Once again, this experiment illustrated the impact that the number of averaged scans had on the frequency of acquiring analytical MS/MS spectra. The combination of $m=$ three full scans and $n=1$ MS/MS scan produced an average of $24.7 \pm 0.6$ analytical MS/MS scan attempts during the one-min time period, while $n=9 \mathrm{MS} / \mathrm{MS}$ scans yielded only $9.3 \pm 0.6$ attempted analytical MS/MS spectra. The quality of these spectra was exhibited in the number of BSA peptides that were identified during the 1-min acquisition time. On average, only $1.0 \pm 0.0 \mathrm{BSA}$ peptides were identified with $m=3$ full scans and $n=1$ MS/MS scan, whereas $3.3 \pm 0.6$ peptides were identified using $n=9$ MS/MS scans. Once again, this demonstrated how the ion statistics were improved by averaging more scans, leading to higher quality spectra. Closer inspection of 
Table 5. Average cross-correlation scores of four unique BSA peptides using 500, 600, and 700-ms MS/MS maximum ion injection times

\begin{tabular}{|c|c|c|c|c|}
\hline & AEFVEVTK $(+1)$ & $\operatorname{LVTDLTK}(+1)$ & HLVDEPQNLIK (+2) & $\begin{array}{l}\text { KVPVOVSTPT } \\
\text { LVEVSR }(+2)\end{array}$ \\
\hline $500 \mathrm{~ms}$ & $1.87 \pm 0.06^{\dagger}$ & $1.52 \pm 0.18^{\dagger}$ & $N / A^{*}$ & $N / A^{*}$ \\
\hline $600 \mathrm{~ms}$ & $2.18 \pm 0.19^{\dagger}$ & $1.67 \pm 0.10^{\dagger}$ & $3.37 \pm 0.24^{\dagger}$ & $3.70 \pm 0.49^{\dagger}$ \\
\hline $700 \mathrm{~ms}$ & $1.53 \pm 0.34^{\dagger}$ & $N / A^{*}$ & $2.80 \pm 0.09^{\dagger}$ & $2.97 \pm 0.46^{\dagger}$ \\
\hline
\end{tabular}

*N/A = peptide was not correctly identified in all replicates.

${ }^{\dagger}$ Relative standard deviation.

the data showed that one or three averaged MS/MS scans produced insufficient spectra quality and led to the identification of $\leq 1$ BSA peptide on average. The greatest enhancement in spectra quality was achieved by increasing the number of averaged MS/MS scans from 3 to 5 , with more subtle increases in quality from
5 to 7 and 7 to 9 averaged MS/MS scans. However, by plotting the number of attempted analytical MS/MS spectra and the number of identified BSA peptides both as a function of averaged MS/MS scans on the same graph (Figure 4e), relationship between the number of acquired MS/MS spectra and spectrum quality became

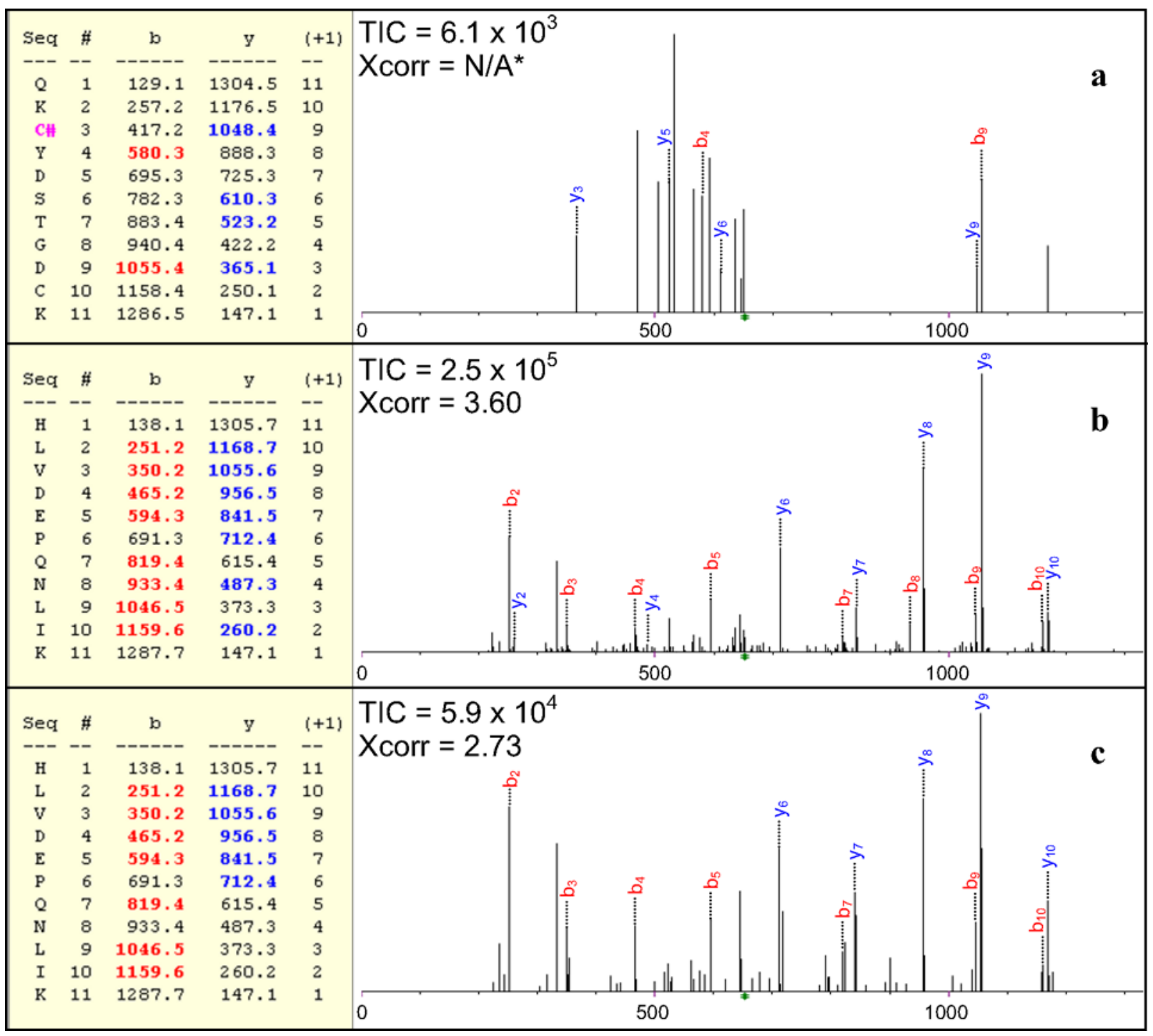

Figure 3. SEQUEST $b$ and $y$ ion assignments for MS/MS spectra of BSA peptide HLVDEPQNLIK $(+2)$. (a) Spectrum acquired with 500-ms maximum ion injection time. (b) Spectrum acquired with 600-ms maximum ion injection time. (c) Spectrum acquired with 700-ms maximum ion injection time. ${ }^{*} \mathrm{~N} / \mathrm{A}=$ amino acid sequence incorrectly identified. 


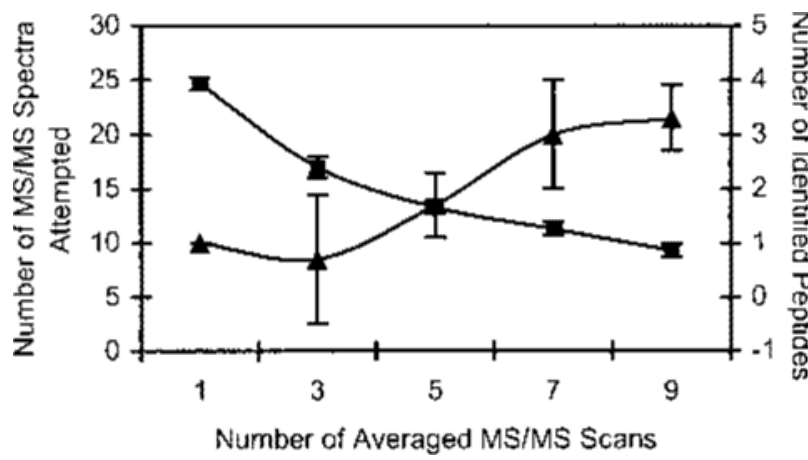

Figure 4. A graph depicting the results of the flow injection experiment using $5 \mu \mathrm{L}$ injections of $10 \mathrm{ng} / \mu \mathrm{L}$ BSA digest. Spectra were acquired in a data-dependent manner for one min. The graph shows the number of analytical MS/MS spectra that were attempted (filled square) and the number of identified BSA peptides (filled triangle) as a function of the number of averaged MS/MS scans.

apparent. Although nine averaged MS/MS scans yielded the greatest number of identified peptides, the time required to assemble one analytical MS/MS spectrum was too long for a typical proteomics analysis of a complex mixture of peptides and would result in missing some coeluting peptides. It became evident that a compromise must be made between the frequency of acquiring analytical MS/MS scans and spectrum quality. The combination of $m=3$ full scans and $n=5$ MS/MS scans seemed to be the logical choice. The 5 averaged MS/MS scans produced spectra that were far superior to that of one or three averaged MS/MS scans while requiring less acquisition time than nine averaged MS/MS scans. One may argue that seven averaged MS/MS scans are even better than 5, but upon inspection of the their respective number of BSA peptides that were cumulatively identified from the three replicate flow injection runs, only one more peptide was identified using seven averaged MS/MS scans than was identified using 5 averaged scans. In light of the need for high spectrum acquisition frequency, this was not a significant enhancement.

These experiments revealed the complexity of optimizing ion trap parameters for the purpose of performing bottom-up proteomics in a data-dependent fashion. It was obvious that one element to achieving high quality MS/MS spectra was the ion abundance in the trap, as was indicated by the ion injection time experiments. Adding to this complexity was the dynamic ion abundance across an eluting peptide peak. The location on the peak at which an MS/MS was performed greatly affected the quality of the MS/MS spectrum and the resulting Xcorr (Figure 5). It would have been desirable to acquire an MS/MS spectrum of an eluting peptide at the apex of its chromatographic peak since this would provide the highest abundance of precursor ions in the trap, thus the highest possible quality of spectrum. Unfortunately, there is currently no way to accomplish this feat.

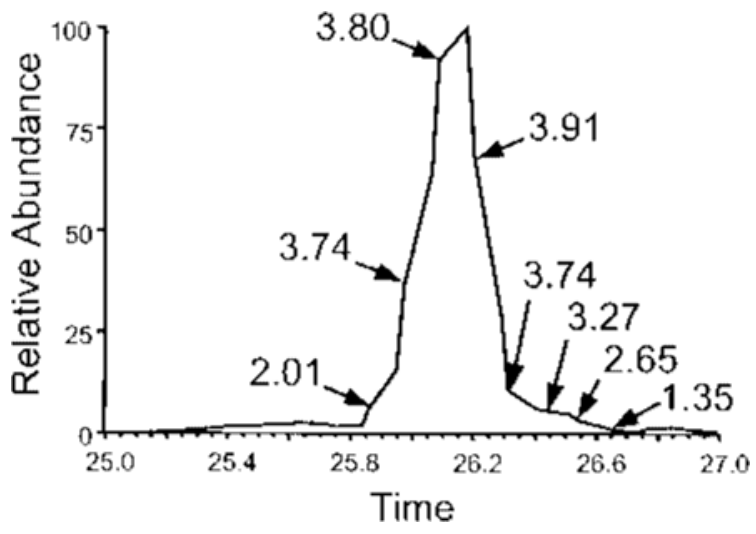

Figure 5. Cross-correlation scores for BSA peptide KVPQVSTPTLVEVSR (+2), $880.8 \mathrm{~m} / \mathrm{z}$. MS/MS spectra were obtained at the denoted locations on the chromatographic peak and subjected to a protein database search with SEQUEST.

\section{Conclusions}

In this paper, we describe a range of ion trap parameters for the Finnigan LCQ Deca ion trap mass spectrometer to enhance the ability to acquire high quality MS/MS spectra for the purpose of proteomics. We have illustrated how achieving high quality spectra requires the simultaneous cooperation of several dynamic elements, including peak capacity, analytical scan acquisition rate, and ion abundance. For example, we have shown how decreasing the number of analytical scans per $s$ by averaging more scans reduces the number of spectra that can be acquired over a given time. However, to achieve high quality spectra amenable to unambiguous peptide correlations, we must average at least $m=1$ full scan and $n=3$ MS/MS scans. On the other hand, when dealing with very complex mixtures of peptides that may generate poorly resolved or coeluting chromatographic peaks, we have determined that no more than 5 full scans and 9 MS/MS scans should be averaged. Beyond this, spectrum quality is not necessarily increased, but rather the rate of acquiring analytical scans becomes too slow and some eluting peptides are not polled. We have also shown that by changing the maximum ion injection time from $500 \mathrm{~ms}$ to $600 \mathrm{~ms}$, the quality of MS/MS spectra is greatly increased, leading to better peptide correlations.

In conclusion, we have determined that to identify a unique set of ion trap parameters applicable to proteomics LC/MS/MS experiments, a compromise must be made between the acquisition frequency of analytical scans and the resulting quality of the MS/MS spectra. For most data-dependent proteomics experiments, we have shown that the following rule of thumb would apply: 3 averaged full scans, 5 averaged MS/MS scans, and a maximum ion injection time of $600 \mathrm{~ms}$. These settings produce MS/MS spectra among the highest possible quality while still maintaining a rate of spectrum acquisition that is capable of analyzing all eluting peptides in complex mixture. 


\section{Acknowledgments}

These authors thank the University of Kentucky Mass Spectrometry Facility for the use of its resources (www.rgs.uky.edu/ ukmsf). They also extend a special thanks to Dr. Jack Goodman for his helpful discussions and suggestions.

\section{References}

1. Peng, J.; Gygi, S. P. Proteomics: The Move to Mixtures. J. Mass. Spectrom. 2001, 36, 1083-1091.

2. Choudhary, G.; Wu, S. L.; Shieh, P.; Hancock, W. S. Multiple Enzymatic Digestion for Enhanced Sequence Coverage of Proteins in Complex Proteomic Mixtures Using Capillary LC with Ion Trap MS/MS. J. Proteome Res. 2003, 2, 59-67.

3. Kuwana, R.; Kasahara, Y.; Fujibayashi, M.; Takamatsu, H.; Ogasawara, N.; Watabe, K. Proteomics Characterization of Novel Spore Proteins of Bacillus subtilis. Microbiology 2002, 148, 3971-3982.

4. Gururaja, T. L.; Li, W.; Payan, D. G.; Anderson, D. C. Utility of Peptide-Protein Affinity Ccomplexes in Proteomics: Identification of Interaction Partners of a Tumor Suppressor Peptide. J. Pept. Res. 2003, 61, 163-176.

5. Foster, L. J.; De Hoog, C. L.; Mann, M. Unbiased Quantitative Proteomics of Lipid Rafts Reveals High Specificity for Signaling Factors. Proc. Natl. Acad. Sci. U.S.A. 2003, 30, 30.

6. Washburn, M. P.; Ulaszek, R.; Deciu, C.; Schieltz, D. M.; Yates, J. R. III. Analysis of Quantitative Proteomic Data Generated via Multidimensional Protein Identification Technology. Anal. Chem. 2002, 74, 1650-1657.

7. Peng, J.; Elias, J. E.; Thoreen, C. C.; Licklider, L. J.; Gygi, S. P. Evaluation of Multidimensional Chromatography Coupled with Tandem Mass Spectrometry (LC/LC-MS/MS) for LargeScale Protein Analysis: The Yeast Proteome. J. Proteome Res. 2003, 2, 43-50.

8. Washburn, M. P.; Wolters, D.; Yates, J. R. III. Large-Scale Analysis of the Yeast Proteome by Multidimensional Protein Identification Technology. Nat. Biotechnol. 2001, 19, 242-247.

9. Wolters, D. A.; Washburn, M. P.; Yates, J. R. III. An Automated Multidimensional Protein Identification Technology for Shotgun Proteomics. Anal. Chem. 2001, 73, 5683-5690.

10. Mawuenyega, K. G.; Kaji, H.; Yamuchi, Y.; Shinkawa, T.; Saito, H.; Taoka, M.; Takahashi, N.; Isobe, T. Large-Scale Identification of Caenorhabditis elegans Proteins by Multidimensional Liquid Chromatography-Tandem Mass Spectrometry. J. Proteome Res. 2003, 2, 23-35.

11. Link, A. J.; Eng, J.; Schieltz, D. M.; Carmack, E.; Mize, G. J.; Morris, D. R.; Garvik, B. M.; Yates, J. R. III. Direct Analysis of
Protein Complexes Using Mass Spectrometry. Nat. Biotechnol. 1999, 17, 676-682.

12. Ostrowski, L. E.; Blackburn, K.; Radde, K. M.; Moyer, M. B.; Schlatzer, D. M.; Moseley, A.; Boucher, R. C. A Proteomic Analysis of Human Cilia: Identification of Novel Components. Mol. Cell. Proteomics 2002, 1, 451-465.

13. Cutillas, P. R.; Norden, A. G.; Cramer, R.; Burlingame, A. L.; Unwin, R. J. Detection and Analysis of Urinary Peptides by on-Line Liquid Chromatography and Mass Spectrometry: Application to Patients with Renal Fanconi Syndrome. Clin. Sci. (Lond.) 2003, 104, 483-490.

14. Wu, S. L.; Amato, H.; Biringer, R.; Choudhary, G.; Shieh, P.; Hancock, W. S. Targeted Proteomics of Low-Level Proteins in Human Plasma by LC/MSn: Using Human Growth Hormone as a Model System. J. Proteome Res. 2002, 1, 459-465.

15. Wang, H.; Hanash, S. Multi-Dimensional Liquid Phase Based Separations in Proteomics. J. Chromatogr. B Anal. Technol. Biomed. Life Sci. 2003, 787, 11-18.

16. Eng, J. K.; McCormack, A. L.; Yates, J. R. III. An Approach to Correlate Tandem Mass Spectral Data of Peptides with Amino Acid Sequences in a Protein Database. J. Am. Soc. Spectrom. 1994, 5, 976-989.

17. Perkins, D. N.; Pappin, D. J.; Creasy, D. M.; Cottrell, J. S. Probability-Based Protein Identification by Searching Sequence Databases Using Mass Spectrometry Data. Electrophoresis 1999, 20, 3551-3567.

18. Opiteck, G. J.; Ramirez, S. M.; Jorgenson, J. W.; Moseley, M. A. III. Comprehensive Two-Dimensional High-Performance Liquid Chromatography for the Isolation of Overexpressed Proteins and Proteome Mapping. Anal. Biochem. 1998, 258, 349 361.

19. Opiteck, G. J.; Jorgenson, J. W. Two-Dimensional SEC/RPLC Coupled to Mass Spectrometry for the Analysis of Peptides. Anal. Chem. 1997, 69, 2283-2291.

20. Link, A. J. Multidimensional Peptide Separations in Proteomics. Trends Biotechnol. 2002, 20, S8-13.

21. Issaq, H. J. The Role of Separation Science in Proteomics Research. Electrophoresis 2001, 22, 3629-3638.

22. Arnott, D. P.; Gowinowicz, M.; Grant, R. A.; Neubert, T. A.; Packman, L. C.; Speicher, K.; Stone, K.; Turck, C. W. Poster R8-W/ABRF-PRG03: Phosphorylation Site Determination. http: / / www.abrf.org/ResearchGroups/Proteomics/EPosters/ ABRF_PRG03.pdf (accessed October 2003).

23. Russell, W. K.; Park, Z. Y.; Russell, D. H. Proteolysis in Mixed Organic-Aqueous Solvent Systems: Applications for Peptide Mass Mapping Using Mass Spectrometry. Anal. Chem. 2001, 73, 2682-2685. 